\title{
Surgical treatment of duodenal adenocarcinoma: ampullary vs. non-ampullary, short- and long-term outcomes
}

\author{
Jana Tesarikova a , Pavel Skalicky a , Daniela Kurfurstova ${ }^{b}$, Hana Svebisovac ${ }^{c}$ Ondrej Urban ${ }^{d}$, Premysl Faltd , Jana Zapletalovae, \\ Dusan Klos ${ }^{\mathrm{a}}$, Martin Lovecek ${ }^{\mathrm{a}}$
}

Background. The aim of this study was to evaluate symptoms, diagnostic methods, short- and long-term outcomes of surgical treatment in patients with duodenal adenocarcinoma.

Patients and Methods. A single center, retrospective, observational study of 52 consecutive patients with duodenal adenocarcinoma operated on with curative intent between 2006 - 2019. Duodenectomy as part of a hemipancreatoduodenectomy or total pancreatectomy procedure was performed for ADAC (ampullary duodenal/intestinal adenocarcinoma) or NADAC (non-ampullary duodenal adenocarcinoma).

Results. Prevailing symptoms were obstructive jaundice in the ADAC group $(P<0.0001)$ and bleeding in the NADAC group $(P=0.005)$, with larger tumor size in patients with NADAC $(P=0.001)$. Complication rate, morbidity and mortality were comparable. Primary total pancreatoduodenectomy predominated in the NADAC group, $16.6 \%$ vs. $2.9 \%$, and salvage completion pancreatectomy in the ADAC group, $6 \%$ vs. $0 \%$. Significant prognostic factors for OS were perineural invasion $(P=0.006)$ and adjuvant chemotherapy $(P=0.045)$ in the ADAC group, and for DFS the total number of resected lymph nodes $(P=0.042)$ and lymph node ratio $(P=0.031)$ in the NADAC group. Median OS is 21 months and 5 -year survival $27.3 \%$ in the NADAC group and 41.5 months and 52\% in the ADAC group.

Conclusion. Ampullary duodenal/intestinal adenocarcinomas are smaller than non-ampullary at diagnosis, with a higher rate of lymph node metastases, but with a better prognosis and long-term outcome in the presented cohort. Oral localisation of NADAC prevailed in the present cohort. Perineural invasion and postoperative oncological therapy are significant prognostic factors for OS in ADAC, but the total number of lymph nodes and lymph node ratio are significant prognostic factors for DFS in NADAC.

Key words: duodenal carcinoma, non-ampullary carcinoma, ampullary carcinoma, outcomes, long-term survival

Received: January 15, 2021; Revised: April 23, 2021; Accepted: April 23, 2021; Available online: May 11, 2021

https://doi.org/10.5507/bp.2021.028

(c) 2022 The Authors; https://creativecommons.org/licenses/by/4.0/

${ }^{a}$ Department of Surgery l, Faculty of Medicine and Dentistry, Palacky University Olomouc and University Hospital Olomouc, Czech Republic ${ }^{b}$ Department of Clinical and Molecular Pathology, Faculty of Medicine and Dentistry, Palacky University Olomouc and University Hospital Olomouc, Czech Republic

'Department of Oncology, Faculty of Medicine and Dentistry, Palacky University Olomouc and University Hospital Olomouc, Czech Republic ${ }^{d}$ Department of Internal Medicine II - Gastroenterology and Geriatrics, Faculty of Medicine and Dentistry, Palacky University Olomouc and University Hospital Olomouc, Czech Republic

${ }^{e}$ Department of Medical Biophysics, Faculty of Medicine and Dentistry, Palacky University Olomouc, Czech Republic Corresponding author: Martin Lovecek, e-mail: martin.lovecek@fnol.cz

\section{INTRODUCTION}

The majority of pancreatoduodenectomies are performed for pancreatic cancers $(34-72 \%)$ followed by ampullary (16-50\%), biliary (5-39\%) and duodenal cancers $(0-25 \%)$ (ref. $\left.{ }^{1}\right)$. Duodenal adenocarcinomas are rare malignant tumors with a low incidence (about $2 \%$ of colorectal carcinomas), but are the most common malignant tumors of the small bowel ${ }^{2,3}$. Duodenal location is observed in $55 \%$ of all small bowel malignancies ${ }^{2,3}$, and about $50 \%$ of small bowel adenocarcinomas are located in the ampullary region ${ }^{4}$. Duodenal carcinomas and intestinal type of ampullary carcinomas ${ }^{5}$ form a logical and therapeutic subgroup of duodenal malignancies ${ }^{1}$. Radical resections achieved by hemipancreatoduodenectomy are performed similarly and are the only potentially curative procedure used for both types of invasive adenocarcinomas. Duodenal carcinomas are considered to be more aggressive forms of carcinomas ${ }^{6}$. Due to their rarity, literary sources are limited.

The aim of this study is a single center retrospective analysis of consecutive patients operated on for duodenal adenocarcinoma (non-ampullary duodenal and ampullary duodenal/intestinal), evaluating symptoms, diagnostic methods and comparing long-term and shortterm outcomes between these duodenal adenocarcinoma subgroups. To the best of our knowledge, this is the first clinical study focused on duodenal cancers (non-ampullary, ampullary duodenal/intestinal only) from the Czech Republic and central Europe. 


\section{MATERIALS AND METHODS}

A single center, retrospective, observational study, included 52 consecutively operated patients with duodenal cancer. The study and data were collected in accordance with the institutional review board (approval reference no. 159/16). Data of patients with non-ampullary duodenal adenocarcinomas (NADAC) and ampullary duodenal/ intestinal adenocarcinomas (ADAC) were extracted from medical records 5 . The exclusion criteria were as follows: suspected invasive tumor of the pancreas, duodenal metastases, suspected invasive tumor of the distal bile duct, histopathological features different than duodenal adenocarcinoma. Collected data including age, body mass index (BMI), tumor size, hospital stay, number of lymph nodes, lymph node ratio, sex, ASA (American anesthesiologists society physical status classification system) score, previous malignancy, grade, perineural invasion (PN), lymphangioinvasion (LI), angioinvasion ( $\mathrm{AI})$, postoperative complications and adjuvant oncological therapy were analyzed as predictive factors for overall survival (OS) and disease free survival (DFS) in both groups. Other evaluated parameters were 30-day mortality, in-hospital mortality, 1-year, 2-year, 3-year, 5-year survival, recurrence, bleeding as first symptom, first type of examination and Kaplan-Meier survival analysis. Survival analysis of resected NADAC patients was performed in 15 (who reached the evaluated interval) of the 18 patients. Three patients were excluded; one died in the postoperative period, in one patient with unexpected liver metastasis the resection was completed due to bleeding as a palliative procedure and one did not yet reach the one-year interval for long-term survival evaluation. Survival analysis of resected ADAC patients was performed on all patients who reached the evaluated interval.

All 52 consecutive patients with preoperatively endoscopically diagnosed duodenal adenocarcinoma - ADAC (34 pts.) and NADAC (18 pts.) were operated on between 2006-2019. Standard pylorus preserving hemipancreatoduodenectomy, Whipple procedure, or total pancreatoduodenectomy with lymphadenectomy were performed with curative intent ${ }^{?}$.

Hematoxylin-and-eosin stained slides were examined from all cases. Ampullary duodenal adenocarcinoma (ADAC) without invasion to the bile duct and pancreas according to pathological examination were classified as ampullary duodenal/intestinal cancer ${ }^{5}$. Non-ampullary duodenal adenocarcinoma (NADAC) without invasion to the bile duct and pancreas and ampulla or with secondary infiltration of the ampulla were classified as duodenal non-ampullary, in the literature sometimes referred to as SNADEN (sporadic non-ampullary duodenal epithelial neoplasia) ( ref. $\left.^{8}\right)$.

Postoperative complications were classified according to the Clavien-Dindo classification ${ }^{9}$, postoperative oncological therapy was administered at the Comprehensive Cancer Center at the University Hospital in Olomouc. Thirty-days, in-hospital mortality, OS from date of surgery to date of death and DFS from date of surgery to date of recurrence were calculated. Since there are no available relevant oncological markers for ADAC and NADAC, the appearence of new liver mass(es), lymph nodes, locoregional mass(es) or peritoneal dissemination on CT or PETCT were considered to be a recurrence. The 7th edition of TNM classification was used for classification of disease stage. OS and DFS were calculated up to the end of 2020 using censored data.

\section{Statistical analysis}

IBM SPSS Statistics ver. 22 was used for statistical analysis. Kaplan-Meier analysis and Log-rank test were used for estimation of mean of survival and median survival. Cox's regression analysis was used for evaluation of predictive factors of overall survival and disease-free survival. A $P$-value equal to or less than 0.05 was considered significant.

\section{RESULTS}

In 2006-2019, 570 hemipancreatoduodenectomies or total pancreatoduodenectomies were performed at the Department of Surgery I, University Hospital Olomouc. Although duodenal carcinomas are considered to be rare, as much as $52(9.1 \%)$ of all duodenectomies performed as a part of hemipancreatoduodenectomy or total pancreatoduodenectomy were on patients with sporadic duodenal adenocarcinomas and ampullary duodenal adenocarcinomas in our cohort. Eighteen (34\%) were non-ampullary (NADAC) and 34 (66\%) were ampullary duodenal/intestinal type (ADAC). None of them had a history of familial adenomatous polyposis.

Statistically significant differences among both groups were found in initial symptoms; bleeding $(P=0.005)$ in NADAC and obstructive jaundice $(P<0.0001)$ in ADAC, and its initial diagnostic procedures; duodenoscopy (NADAC) and ERCP (ADAC). Tumor size from the resected specimens differed; median size in the ADAC group was $16.5 \mathrm{~mm}$ vs. $35.0 \mathrm{~mm}$ in NADAC $(P=0.001)$. Lymph node metastases were identified in $52.9 \%$ of ADAC and $27.7 \%$ of NADAC. Male sex was more frequent in the ampullary duodenal/intestinal carcinoma group, but was statistically not significant $(P=0.071)$. There were no differences among both groups in the following categories: age, BMI, hospital stay, total number of dissected lymph nodes, number of positive lymph nodes and lymph node ratio, ASA, previous malignancy, grade, perineural invasion, lymphovascular invasion, angioinvasion, postoperative complications and administration of postoperative chemotherapy - Table 1. Primary total pancreatoduodenectomy (TP) was performed in $16.6 \%$ of NADAC vs. $2.9 \%$ in ADAC. The indication for primary TP was made by the surgeon in cases of extremely soft pancreas. Thus, the rate of pancreatic fistula is different, $21.2 \%$ in the ADAC group vs. $0 \%$ in the NADAC group. Table 2 displays parameters with a statistically significant effect on overall survival and Table 3 displays parameters influencing disease-free survival among both 
Table 1. Demographic, clinical and procedural characteristics of the study population.

\begin{tabular}{lccc}
\hline Characteristics & ADAC & NADAC & \\
\hline Duodenal adenocarcinoma, 52 total & $34(65.4 \%)$ & $18(34.6 \%)$ & 0.729 \\
Age, y, median (range) & $63.5(47.0-79.0)$ & $63.0(45.0-82.0)$ & 0.071 \\
Male gender & $79.4 \%$ & $55.6 \%$ & 0.471 \\
BMI & $26.7(18.0-35.4)$ & $25.5(17.5-38.5)$ & $\mathbf{0 . 0 0 5}$ \\
Bleeding & $2.9 \%$ & $33.3 \%$ & $\mathbf{0 . 0 0 0 1}$ \\
Obstructive jaundice & $73.5 \%$ & 0 & $\mathrm{NA}$ \\
Duodenal obstruction & 0 & $5.5 \%$ & $\mathbf{0 . 0 0 1}$ \\
Tumor size, median (mm) & 16.5 & 35.0 & 0.818 \\
Lymph node metastases & $52.9 \%$ & $27.7 \%$ & $\mathrm{NA}$ \\
Oral localisation & $\mathrm{NA}$ & $61 \%$ & 0.346 \\
30-day mortality & 0 & $1(5.6 \%)$ & 0.405 \\
90-day mortality & 0 & $1(5.6 \%)$ & 0.405 \\
In-hospital mortality & 0 & $1(5.6 \%)$ & 1.181 \\
Hospital stay & 15.0 & $15.0(9.0-22.0)$ & 0.086 \\
Postoperative pancreatic fistula & $21.2 \%$ & $0 \%$ & 0.297 \\
Delayed gastric emptying & $26.4 \%$ & $11.8 \%$ & 0.654 \\
Postpancreatectomy haemorrhage & $11.7 \%$ & $5.9 \%$ & 1 \\
CD I-IIIa (mild complications) & $47 \%$ & $44.4 \%$ & 1 \\
CD IIIb-IV (severe complications) & $14.8 \%$ & $11.7 \%$ & 0.200 \\
Total pancreatoduodenectomy & $2.9 \%$ & $16.6 \%$ & $\mathrm{NA}$ \\
Completed pancreatectomy & $6 \%$ & $0 \%$ & \\
\hline
\end{tabular}

ADAC, ampulary duodenal/intestinal adenocarcinoma; NADAC, non-ampullary duodenal adenocarcinoma; BMI, body mass index; CD, ClavienDindo classification of surgical complications.

Table 2. Overall survival (OS) and parameters in NADAC and ADAC.

\begin{tabular}{|c|c|c|c|c|c|c|c|c|}
\hline & \multirow{2}{*}{\multicolumn{4}{|c|}{$\begin{array}{c}\text { NADAC } \\
95 \% \text { CI for RR }\end{array}$}} & \multirow{2}{*}{\multicolumn{4}{|c|}{$\begin{array}{c}\text { ADAC } \\
\text { 95\% CI for RR }\end{array}$}} \\
\hline & & & & & & & & \\
\hline & Sig. & $\mathrm{RR}$ & Lower & Upper & Sig. & $\mathrm{RR}$ & Lower & Upper \\
\hline Age & 0.894 & 0.996 & 0.943 & 1.052 & 0.703 & 1.011 & 0.956 & 1.069 \\
\hline Male sex & 0.112 & 2.727 & 0.791 & 9.403 & 0.787 & 0.839 & 0.235 & 2.996 \\
\hline BMI & 0.051 & 0.849 & 0.720 & 1.001 & 0.091 & 0.775 & 0.577 & 1.041 \\
\hline ASA & 0.072 & 3.415 & 0.897 & 13.0 & 0.306 & 1.684 & 0.620 & 4.570 \\
\hline Grade & 0.890 & 1.064 & 0.445 & 2.540 & 0.913 & 1.055 & 0.408 & 2.723 \\
\hline PNI & 0.559 & 1.733 & 0.274 & 11.0 & 0.006 & 5.664 & 1.647 & 19.478 \\
\hline LI & 0.829 & 1.310 & 0.113 & 15.2 & 0.461 & 1.477 & 0.524 & 4.164 \\
\hline AI & & & - & & 0.142 & 5.500 & 0.566 & 53.499 \\
\hline Adjuvancy & 0.570 & 1.455 & 0.400 & 5.297 & 0.045 & 0.330 & 0.111 & 0.976 \\
\hline Total LN & 0.764 & 0.984 & 0.886 & 1.093 & 0.157 & 1.134 & 0.953 & 1.351 \\
\hline Positive LN & 0.126 & 1.294 & 0.930 & 1.800 & 0.560 & 1.174 & 0.684 & 2.015 \\
\hline LNR & 0.059 & 54 & 0.9 & 3444 & 0.472 & 3.646 & 0.108 & 123.559 \\
\hline
\end{tabular}

ADAC, ampullary duodenal/intestinal adenocarcinoma; NADAC, non-ampullary duodenal adenocarcinoma; OS, overall survival; BMI, body mass index; ASA, American society of anesthesiologists physical status classification system; PNI, perineural invasion; LI, lymphangioinvasion; AI, angioinvasion; LN, lymph nodes; LNR, lymph node ratio; CI for RR, confidence interval for risk ratio.

groups - NADAC and ADAC. Grade, perineural invasion, lymphangioinvasion, angioinvasion, total number of lymph nodes, LNR and postoperative chemotherapy were not found to be statistically significant prognostic parameters for overall survival in the NADAC group, but perineural invasion $(P=0.006)$ and administration of adjuvant chemotherapy ( $P=0.045)$ were found to be significant in the group of ADAC (Table 2). For specific disease-free survival, the total number of examined LN ( $P=0.042)$ and LNR $(P=0.031)$ were found to be significant predictive factors in NADAC. BMI $(P=0.051)$, perineural invasion $(P=0.082)$ and adjuvant chemotherapy $(P=0.073)$ were borderline significant in the ADAC group and BMI $(P=0.091)$ in NADAC (Table 3$)$. The survival analysis using Kaplan-Meier analysis shows the differences between survival in both groups, but was only borderline signifi- 
Table 3. Disease-free survival (DFS) and parameters in NADAC and ADAC.

\begin{tabular}{|c|c|c|c|c|c|c|c|c|}
\hline & \multicolumn{4}{|c|}{ NADAC } & \multicolumn{4}{|c|}{ ADAC } \\
\hline & \multicolumn{4}{|c|}{ 95\% CI for RR } & \multicolumn{4}{|c|}{$95 \%$ CI for RR } \\
\hline & Sig. & RR & Lower & Upper & Sig. & RR & Lower & Upper \\
\hline Grade & 0.801 & 1.178 & 0.330 & 4.208 & 0.523 & 0.571 & 0.102 & 3.187 \\
\hline PNI & 0.381 & 3.464 & 0.215 & 55.8 & 0.082 & 8.607 & 0.761 & 97.3 \\
\hline LI & 0.393 & 58.761 & 0.005 & 671566 & 0.685 & 0.635 & 0.071 & 6 \\
\hline AI & \multicolumn{4}{|c|}{-} & 0.808 & & - & \\
\hline Total no of LN & 0.042 & 1.575 & 1.016 & 3.442 & 0.290 & 1.582 & 0.676 & 3.700 \\
\hline LNR & 0.031 & 233 & 1.67 & 32475 & 0.384 & 20 & 0.02 & 16784 \\
\hline Adjuvancy & 0.808 & 1.227 & 0.235 & 6.393 & 0.073 & 0.134 & 0.015 & 1.209 \\
\hline
\end{tabular}

DFS, disease free survival; ADAC, ampullary duodenal/intestinal adenocarcinoma; NADAC, non-ampullary duodenal adenocarcinoma; CI for RR, confidence interval for risk ratio; PNI, perineural invasion; LI, lymphangioinvasion; AI, angioinvasion; LN, lymph nodes; LNR, lymph node ratio.

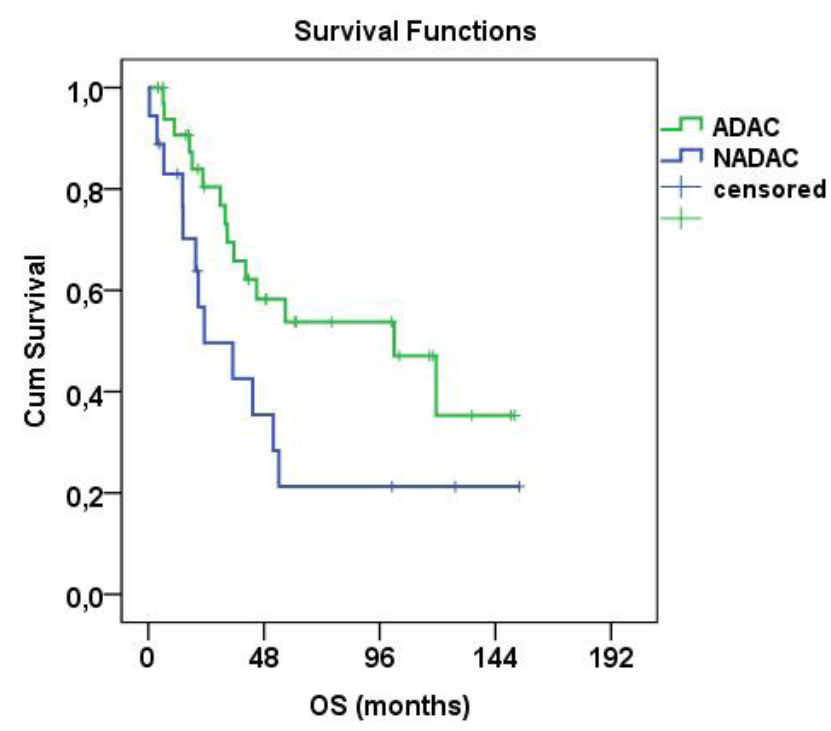

Fig. 1. Kaplan-Meier survival analysis of ADAC vs. NADAC $(\mathrm{P}=0.051)$.

ADAC, ampullary duodenal/intestinal adenocarcinoma; NADAC, nonampullary duodenal adenocarcinoma; OS, overall survival.

cant $(P=0.051)$. The adjuvant chemotherapy regimens used were DDP + 5FU for ADAC, and 5-FU or FUFA regimens for NADAC patients.

\section{Survival analysis}

The 1-year, 2-year, 3-year and 5-year OS rate of NADAC patients after radical resection was $93.3 \%, 57.1 \%$, $50.0 \%$ and $27.3 \%$ respectively, with median follow up 22 months, (range 12-156 months). Lymph node infiltration was found in $27.7 \%$. Mean of DFS in NADAC group was 35.9 months with range 3-154 months, median was 11.7 months. The locations of reccurence were equaly represented in the liver (2), retroperitoneal lymph nodes (2) and peritoneum (2); in 2 were not reported exactly.

Survival analysis of resected ADAC patients was performed on all patients who reached the evaluated interval. The 1-year, 2-year, 3-year and 5-year OS rate after radical resection was $93.5 \%, 78.6 \%, 64.3 \%$ and $52 \%$ respectively, with median follow up 41 months, (range 4-154 months). Lymph node infiltration was found in $52.9 \%$. Mean of DFS in ADAC groups was 62.9 months with range 3-152 months, median was 53.1 months. The location of recurrence was mainly the liver (4) or liver and lymph nodes (1), in six were not clearly reported.

\section{DISCUSSION}

This study focuses on potentially curative surgical treatment of duodenal adenocarcinomas. Duodenal carcinomas are more aggressive gastrointestinal tumors, but with a better prognosis than pancreatic carcinomas ${ }^{10}$, and with the most favourable survival among periampullary cancer patients following resection ${ }^{11}$. The potentially curative treatment procedure of invasive carcinomas in that region is the same - hemipancreatoduodenectomy or total pancreatoduodenectomy. According to their relationship with the papilla of Vater, logically duodenal cancers may be divided into a) ampullary duodenal/intestinal adenocarcinomas (ADAC) and b) non-ampullary duodenal adenocarcinomas (NADAC) ( ref. $^{1}{ }^{1}$ ). Ampullary carcinomas are located in the transitional region. They present as very heterogenous tumors with hybrid phenotypes consisting of pancreatobiliary and intestinal epithelium and cause subjectivity in their histological classification $^{12}$. Morphologic classification of ADAC is based on the predominant pattern: pancreatobiliary or intestinal, and it correlates with prognosis ${ }^{12}$. In the present study, ADAC inclusion criteria included the absence of infiltration of the pancreas or pancreatic duct and bile duct. Non-ampullary duodenal adenocarcinomas according to morphological studies ${ }^{13}$ can be different; gastric type, intestinal and less frequently pancreatobiliary type, with more aggressive behaviour among gastric/pancreatobiliary types ${ }^{10,13,14}$. The true incidence of non-ampullary and ampullary duodenal/intestinal adenocarcinomas is not clearly known. The Czech National Oncological Registry shows that the incidence of duodenal malignancies was $0.91 / 100.000$ in 2012, with a two-fold increase in the last 40 years $^{15}$. The incidence of non-ampullary duodenal 
carcinoma according to the most recent and only study stated the incidence as 2.3/100.000 in the Japanese population $^{16}$ and 5.4/1.000.000 in Denmark ${ }^{17}$.

In the presented single center cohort, 52 duodenal adenocarcinomas were operated on with curative intent over 14 years. ADAC was identified in $65.4 \%$ and NADAC in $34.6 \%$. The haemorrhage is the dominating initial symptom $(P=0.005)$ and endoscopy $(P<0.0001)$ is the first diagnostic tool in the group of NADAC, and obstructive jaundice and ERCP $(P<0.0001)$ in the group of ADAC.

Matsueda et al. ${ }^{7}$ in their multi-institutional analysis found that orally localised non-ampullary duodenal carcinomas have different clinicopathological features and a more aggressive behaviour with higher recurrence rate and shorter DFS than aborally localised carcinomas ${ }^{10,13}$. The presented study was unable to prove a difference in survival in the NADAC group based on the location, but more than $60 \%$ of all NADAC tumors were localised completely in the oral part of duodenum and $71 \%$ of the rest of NADAC tumors presented an oral component. The 5 -year survival was worse $(27.3 \%$ vs. $57 \%)$ than previously published studies ${ }^{10}$. In a large review by Cloyd et al. (ref. ${ }^{6}$ ), 24 studies with duodenal non-ampullary carcinomas were reviewed. One to 132 patients were treated with pancreatoduodenectomy in this review. In terms of radicality, hemipancreatoduodenectomy is a more favorable resection method than segmental resection, due to greater radicality with a higher percentage of $\mathrm{R} 0$ resections and a more sufficient lymphadenectomy than in segmental resections. The extent of the lymphadenectomy and the number of harvested LNs is one of the prognostic factors in duodenal adenocarcinoma ${ }^{6}$ and number of lymph node metastases is the independent prognostic factor among ADAC and NADAC (ref. ${ }^{14,18}$ ). Lymph node invasion (Nitta et al. ${ }^{19}$ ) and lymphovascular invasion were independent prognostic factors in Solaini's study from the UK $\left(\right.$ ref. $\left.^{20}\right)$. All patients in the cohort were treated with duodenectomy as part of a hemipancreatoduodenectomy or total pancreatoduodendectomy procedure with standard lymphadenectomy ${ }^{7}$. Lymph node infiltration was found in $27.7 \%$ of NADAC vs. $52.9 \%$ in ADAC group. Only the total number of harvested LNs $(P=0.042)$ and LNR $(P=0.031)$ were significant prognostic factors for DFS in the group of NADAC. Regarding tumor size, NADAC (35.0 mm) were significantly larger then ADAC $(16.5 \mathrm{~mm})(P=0.001)$. This is a completely inverse finding when compared with Xue et al. (ref. ${ }^{10}$ ). Tumor characteristics like grade, vascular invasion, lymphangioinvasion were not proved to be prognostic factors in both groups, but perineural invasion was a stastically singificant factor for OS in the group of $\operatorname{ADAC}(P=0.006)$. Pancreatic invasion is a newly studied prognostic factor in duodenal carcinoma, and Nitta et al. in their latest study found pancreatic invasion to be an independent prognostic factor ${ }^{19}$. Pancreatic resections are associated with significant morbidity and mortality, mostly caused by postoperative pancreatic fistula (POPF) and its complications. According to Malleo et al. postoperative complications may be an additional prognostic factor ${ }^{21}$. Specific postoperative complications like POPF and postpancreatectomy haemor- rhage $(\mathrm{PPH})$ were more frequent in the group of ADAC vs. NADAC; POPF $21.1 \%$ vs. $0 \%$, PPH $11.7 \%$ vs. $5.9 \%$; secondary salvage pancreatectomy was performed in $6 \%$ vs. $0 \%$. According to the surgeon's subjective decision, based on the softness of the pancreatic parenchyma and in order to reduce severe complications, total pancreatoduodenectomy was performed more frequently in the NADAC group; $16.6 \%$ vs. $2.9 \%$ in the ADAC group. Complication rate and hospital stay were equal in both subgroups, but due to the higher rate of total pancreatectomies, lower rates of POPF, DGE and PPH were seen in the group of NADAC. Total pancreatectomy is considered to be a safe and reasonable surgical procedure with excellent perioperative morbidity and mortality in pancreatic cancer ${ }^{22}$. In our set of duodenal carcinomas total pancreatectomy in cases of soft pancreas is considered to be safe with reasonable morbidity and mortality.

\section{Long term outcomes}

According to the literature, median survival in non-resectable and metastatic duodenal cancer is $2-8$ months ${ }^{23-26}$. In patients with resectable duodenal cancer, the long-term outcome - OS - is longer than with other periampullary cancers (pancreatic, biliary). A radical surgical procedure is mandatory for long-term survival. Sohn and Poulsides reported more than 50\% 5-year OS when R0 was achieved vs. 0\% 5-year OS for R1 resections $^{27,28}$. In the presented study, all procedures were R0 and actual median OS of NADAC has been 21 months and 1-, 3- and 5-year survival rates were 93.3\%, 50.0\% and $27.3 \%$, but oral location, which is considered to be more aggressive, prevailed. Mann et al. reported comparable 1- and 3-year OS among NADAC - 84\% and 52\% $\left(\right.$ ref. $^{28}$ ). In the ADAC group, median OS is 41.5 months and 1-,3- and 5-year survival rates were 93.5\%, 64.3\% and $52 \%$. When compared with Xue's study, 5-year survival in the ADAC in our cohort is comparable; $52 \%$ vs. $52 \%$, but in the NADAC group is worse; $27.3 \%$ vs. $57 \%$ (ref. ${ }^{10}$ ). Mann et al. reported 1- and 3-year OS among NADAC $84 \%$ and $52 \%\left(\right.$ ref. $^{28}$ ).

\section{CONCLUSION}

In conclusion, duodenal adenocarcinomas, ampullary/ intestinal and non-ampullary are the smallest group of carcinomas treated with radical hemipancreatoduodenectomy or total pancreatoduodenectomy, but have a more favourable prognosis than other periampullary carcinomas (pancreatic, distal bile duct). Only radical surgery offers a chance of longer survival. Ampullary duodenal/intestinal cancers are diagnosed smaller than non-ampullary, with a higher rate of lymph node metastases, but with a better prognosis and long-term outcomes in the presented cohort. Oral location of non-ampullary cancers is associated with worse OS. Perineural invasion and postoperative oncological therapy are significant prognostic factors for $\mathrm{OS}$ in the group of ADAC, but the total number of lymph nodes and lymph node ratio are most significant for DFS in the group of NADAC. 


\section{ABBREVIATIONS}

ADAC, Ampullary duodenal/intestinal adenocarcinoma; NADAC, Non-ampullary duodenal adenocarcinoma; OS, Overall survival; DFS, Disease free survival; LN, Lymph nodes; LNR, Lymph node ratio; BMI, Body mass index; ASA, American society of anesthesiologists physical status classification system; PN, Perineural invasion; LI, Lymphangioinvasion; AI, Angioinvasion; SNADEN, Sporadic non-ampullary duodenal epithelial neoplasia; CD, Clavien Dindo classification; ERCP, Endoscopic retrograde cholangio-pancreatography; TP, Total pancreatoduodenectomy; POPF, Postoperative pancreatic fistula; PPH, Postpancreatectomy haemorhage; DGE, Delayed gastric emptying; DDP + 5FU, Cisdiamminedichloroplatinum + 5-fluorouracil; 5-FU, 5-fluorouracil; FUFA, 5-fluorouracil and folinic acid.

Acknowledgments: Supported by Ministry of Health of Czech Republic, NV19-09-00088 and DRO MEX FN Olomouc 00098892 (87-94).

Author contributions: JT, ML: research design; ML, PS: performed the procedures; JT, PS, DK, HS, OU, PF, JZ: data analysis; JT, DK, ML: manuscript writing; All authors critically revised the manuscript for important intellectual content.

Conflict of interest: The authors state that there are no conflicts of interest regarding the publication of this article.

\section{REFFERENCES}

1. Chandrasegaram MD, Gill AJ, Samra J, Price T, Chen J, Fawcett J, Merrett ND. Ampullary cancer of intestinal origin and duodenal cancer - A logical clinical and therapeutic subgroup in periampullary cancer. World J Gastrointest Oncol 2017;9:407-15. doi: 10.4251/ wjgo.v9.i10.407

2. Zenali M, Overman MJ, Rashid A, Broaddus RB, Wang $H$, Katz MH, Fleming JB, Abbruzzese JL, Wang H. Clinicopathologic features and prognosis of duodenal adenocarcinoma and comparison with ampullary and pancreatic ductal adenocarcinoma. Hum Pathol 2013;44:2792-8. doi: 10.1016/j.humpath.2013.07.030

3. Bal A, Joshi K, Vaiphei K, Wig JD. Primary duodenal neoplasms: a retrospective clinico-pathological analysis. World J Gastroenterol 2007;13:1108-11. doi: 10.3748/wjg.v13.i7.1108

4. Reghunath A, Kabilan K, Mittal MK. Exploring the neglected segment of the intestine: the duodenum and its pathologies. Pol J Radiol 2020;85:e230-e244. doi: 10.5114/pjr.2020.95477. PMID: 32612721; PMCID: PMC7315055.

5. Adsay V, Ohike N, Tajiri T, Kim GE, Krasinskas A, Balci S, Bagci P, Basturk O, Bandyopadhyay S, Jang KT, Kooby DA, Maithel SK, Sarmiento J, Staley CA, Gonzalez RS, Kong SY, Goodman M. Ampullary region carcinomas: definition and site specific classification with delineation of four clinicopathologically and prognostically distinct subsets in an analysis of 249 cases. Am J Surg Pathol 2012;36:1592-608. doi: 10.1097/PAS.0b013e31826399d8

6. Cloyd JM, George E, Visser BC. Duodenal adenocarcinoma: Advances in diagnosis and surgical management. World J Gastrointest Surg 2016;8:212-21. doi: 10.4240/wjgs.v8.i3.212

7. Tol JA, Gouma DJ, Bassi C, Dervenis C, Montorsi M, Adham M, Andrén-Sandberg A, Asbun HJ, Bockhorn M, Büchler MW, Conlon KC, Fernández-Cruz L, Fingerhut A, Friess $\mathrm{H}$, Hartwig W, Izbicki JR, Lillemoe KD, Milicevic MN, Neoptolemos JP, Shrikhande SV, Vollmer CM, Yeo CJ, Charnley RM; International Study Group on Pancreatic Surgery. Definition of a standard lymphadenectomy in surgery for pancreatic ductal adenocarcinoma: a consensus statement by the International Study Group on Pancreatic Surgery (ISGPS). Surgery 2014;156:591-600.

8. Matsueda K, Kanzaki H, Matsueda K, Nasu J, Yoshioka M, Nakagawa M, Inoue M, Inaba T, Imagawa A, Takatani M, Takenaka R, Suzuki S, Tomoda J, Yagi T, Fujiwara T, Tanaka T, Okada H. The clinicopathological differences of sporadic non-ampullary duodenal epithelial neoplasm depending on tumor location. J Gastroenterol Hepatol 2019;34:1540-1544. doi: 10.1111/jgh.14640

9. Clavien PA, Barkun J, de Oliveira ML, Vauthey JN, Dindo D, Schulick RD, de Santibañes E, Pekolj J, Slankamenac K, Bassi C, Graf R, Vonlanthen R, Padbury R, Cameron JL, Makuuchi M. The ClavienDindo classification of surgical complications: five-year experience. Ann Surg 2009;250:187-96. doi: 10.1097/SLA.0b013e3181b13ca2

10. Xue Y, Vanoli A, Balci S, Reid MM, Saka B, Bagci P, Memis B, Choi H, Ohike N, Tajiri T, Muraki T, Quigley B, El-Rayes BF, Shaib W, Kooby D, Sarmiento J, Maithel SK, Knight JH, Goodman M, Krasinskas AM, Adsay V. Non-ampullary-duodenal carcinomas: clinicopathologic analysis of 47 cases and comparison with ampullary and pancreatic adenocarcinomas. Mod Pathol 2017;30:255-266. doi: 10.1038/modpathol.2016.174

11. Tol JA, Brosens LA, van Dieren S, van Gulik TM, Busch OR, Besselink MG, Gouma DJ. Impact of lymph node ratio on survival in patients with pancreatic and periampullary cancer. Br J Surg 2015;102:23745. doi: 10.1002/bjs.9709

12. Reid MD, Balci S, Ohike N, Xue Y, Kim GE, Tajiri T, Memis B, Coban I, Dolgun A, Krasinskas AM, Basturk O, Kooby DA, Sarmiento JM, Maithel SK, El-Rayes BF, Adsay V. Ampullary carcinoma is often of mixed or hybrid histologic type: an analysis of reproducibility and clinical relevance of classification as pancreatobiliary versus intestinal in 232 cases. Mod Pathol 2016;29:1575-1585. doi: 10.1038/ modpathol.2016.124

13. Ushiku T, Arnason T, Fukayama M, Lauwers GY. Extra-ampullary duodenal adenocarcinoma. Am J Surg Pathol 2014;38:1484-93. doi: 10.1097/PAS.0000000000000278

14. Liu N, Liang $H$, Zhang RP, Pan Y, Liu Y, Deng JY, Wang XN, Ding XW, Hao XS. Number of lymph node metastases: a significant prognostic factor for patients with radical resection of carcinoma of the ampulla of Vater. Zhonghua Wei Chang Wai Ke Za Zhi 2007;10:350-2.

15. Ryska M, Hrabal P. Malignang tumors of duodenum. Rozhl Chir 2015;94:497-503.

16. Yoshida M, Yabuuchi Y, Kakushima N, Kato M, Iguchi M, Yamamoto Y, Kanetaka K, Uraoka T, Fujishiro M, Sho M; Japan Duodenal Cancer Guideline Committee. The incidence of non-ampullary duodenal cancer in Japan: The first analysis of a national cancer registry. J Gastroenterol Hepatol 2020. doi: 10.1111/jgh.15285 Epub ahead of print

17. Buchbjerg T, Fristrup C, Mortensen MB. The incidence and prognosis of true duodenal carcinomas. Surg Oncol 2015;24:110-6. doi: 10.1016/j.suronc.2015.04.004

18. Jiang QL, Huang XH, Chen YT, Zhang JW, Wang CF. Prognostic Factors and Clinical Characteristics of Patients with Primary Duodenal Adenocarcinoma: A Single-Center Experience from China. Biomed Res Int 2016;2016:6491049. doi: 10.1155/2016/6491049

19. Nitta N, Ohgi K, Sugiura T, Okamura Y, Ito T, Yamamoto $Y$, Ashida R, Sasaki K, Uesaka K. Prognostic Impact of Pancreatic Invasion in Duodenal Carcinoma: A Single-Center Experience. Ann Surg Oncol 2020;27:4553-560. doi: 10.1245/s10434-020-08512-8

20. Solaini L, Jamieson NB, Metcalfe M, Abu Hilal M, Soonawalla Z, Davidson BR, McKay C, Kocher HM; UK Duodenal Cancer Study Group. Outcome after surgical resection for duodenal adenocarcinoma in the UK. Br J Surg 2015;102:676-81. doi: 10.1002/bjs.9791

21. Malleo G, Tonsi A, Marchegiani G, Casarotto A, Paiella S, Butturini $G$, Salvia R, Bassi C. Postoperative morbidity is an additional prognostic factor after potentially curative pancreaticoduodenectomy for primary duodenal adenocarcinoma. Langenbecks Arch Surg 2013;398:287-94. doi: 10.1007/s00423-012-0978-9

22. Kulu Y, Schmied BM, Werner J, Muselli P, Büchler MW, Schmidt J. Total pancreatectomy for pancreatic cancer: indications and operative technique. HPB (Oxford) 2009;11:469-75. doi: 10.1111/j.14772574.2009.00085.x

23. Overman MJ, Kopetz S, Wen S, Hoff PM, Fogelman D, Morris J, Abbruzzese JL, Ajani JA, Wolff RA. Chemotherapy with 5-fluorouracil and a platinum compound improves outcomes in metastatic small 
bowel adenocarcinoma. Cancer 2008;113:2038-45. doi: 10.1002/ cncr.23822

24. Overman MJ, Varadhachary GR, Kopetz S, Adinin R, Lin E, Morris JS, Eng C, Abbruzzese JL, Wolff RA. Phase Il study of capecitabine and oxaliplatin for advanced adenocarcinoma of the small bowel and ampulla of Vater. J Clin Oncol 2009;27:2598-603. doi: 10.1200/ JCO.2008.19.7145

25. Lee HG, You DD, Paik KY, Heo JS, Choi SH, Choi DW. Prognostic factors for primary duodenal adenocarcinoma. World J Surg 2008;32:224652.

26. Gibson MK, Holcroft CA, Kvols LK, Haller D. Phase II study of 5-fluorouracil, doxorubicin, and mitomycin $\mathrm{C}$ for metastatic small bowel adenocarcinoma. Oncologist 2005;10:132-37.
27. Sohn TA, Lillemoe KD, Cameron JL, Pitt HA, Kaufman HS, Hruban $\mathrm{RH}$, Yeo CJ. Adenocarcinoma of the duodenum: factors influencing long-term survival. J Gastrointest Surg 1998;2:79-87. doi: 10.1016/ s1091-255x(98)80107-8

28. Poultsides GA, Huang LC, Cameron JL, Tuli R, Lan L, Hruban RH, Pawlik TM, Herman JM, Edil BH, Ahuja N, Choti MA, Wolfgang CL, Schulick RD. Duodenal adenocarcinoma: clinicopathologic analysis and implications for treatment. Ann Surg Oncol 2012;19:1928-35. doi: 10.1245/s10434-011-2168-3

29. Mann K, Gilbert T, Cicconi S, Jackson R, Whelan P, Campbell F, Halloran C, Neoptolemos J, Ghaneh P. Tumour stage and resection margin status are independent survival factors following partial pancreatoduodenectomy for duodenal adenocarcinoma. Langenbecks Arch Surg 2019;404:439-49. doi: 10.1007/s00423-019-01779-w 